\title{
Experimental Mistuning Identification in Bladed Disks Using a Component-Mode-Based Reduced-Order Model
}

\author{
John A. Judge* \\ Catholic University of America, Washington, D.C. 20064 \\ Christophe Pierre \\ McGill University, Montreal, Quebec H3A 2K6, Canada \\ and \\ Steven L. Ceccio \\ University of Michigan, Ann Arbor, Michigan 48109 \\ DOI: $\underline{10.2514 / 1.41214}$
}

\begin{abstract}
This paper presents two methods for identifying blade mistuning using measurements of the vibration response of an entire bladed disk. These methods are especially suitable to blisks, in which individual blades cannot be easily isolated. The first method makes use of measurements of the free response normal modes of the system, whereas the second is an extension of the first to make use of steady-state forced response measurements. For both methods, the variations in blade modal stiffness (the natural frequencies of the blades themselves) are calculated by converting a small set of measured blade vibration amplitudes into coordinates in a highly reduced-order model in which the blade modal stiffnesses appear explicitly. The effectiveness of both methods is demonstrated experimentally.
\end{abstract}

\section{Nomenclature}

$\mathbf{C}$

$=$ viscous damping matrix

$=$ real-values Fourier matrix

$=$ identity matrix

$=$ stiffness matrix

$=$ mass matrix synthesis model

$=$ physical coordinates

$=$ error magnification factor

$=$ variation in blade stiffness

$=$ damping ratio reduced stiffness matrix

$=$ eigenvalue (modal stiffness) $=\left[-\omega^{2} \mathbf{M}+\mathbf{K}\right]$ or $\left[-\omega^{2} \mathbf{M}+i \omega \mathbf{C}+(1+i G) \mathbf{K}\right]$

$=$ structural damping coefficient

$=$ number of sectors in bladed disk

$=$ number of harmonics, int $[N / 2]$

$=$ coordinates in Craig-Bampton component mode

$=$ secondary modal analysis coordinates

$=$ transformation matrix for component assembly

$=$ transformation matrix for change of coordinates

$=$ cyclic disk coordinates

$=$ combined transformation matrix for component mode synthesis and secondary modal analysis

$=$ matrix of secondary modal analysis mode shapes

$=$ secondary modal analysis mode shape vector

$=$ individual component mode synthesis component

$=$ diagonal matrix of eigenvalues (modal stiffness matrix)

$=$ individual component mode synthesis component reduced mass matrix
Received 24 September 2008; accepted for publication 6 January 2009. Copyright $\odot 2009$ by the American Institute of Aeronautics and Astronautics, Inc. All rights reserved. Copies of this paper may be made for personal or internal use, on condition that the copier pay the $\$ 10.00$ per-copy fee to the Copyright Clearance Center, Inc., 222 Rosewood Drive, Danvers, MA 01923; include the code 0001-1452/09 \$10.00 in correspondence with the CCC.

*Assistant Professor, Department of Mechanical Engineering, 620 Michigan Avenue Northeast; judge@ cua.edu.

Dean, Faculty of Engineering, 817 Sherbrooke Street; christophe.pierre@ mcgill.ca. Senior Member AIAA.

Professor, Department of Mechanical Engineering, 2350 Hayward Street; ceccio@umich.edu.

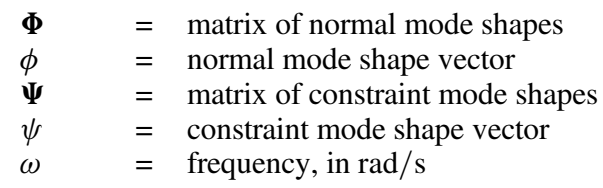

Subscripts

$B=$ partition for interior physical blade degrees of freedom

$b_{m}=$ partition for mistuned blade modal coordinates

$b_{t}=$ partition for tuned blade modal coordinates

$c=$ partition for constraint mode coordinates

$d \quad=$ partition for disk modal coordinates

$e \quad=$ partition corresponding to all coordinates excluding the mistuned blade coordinates

$m=$ secondary modal analysis model partition for mistuned blade coordinates

meas = vector/matrix subset corresponding to measured degrees of freedom

$S=$ partition for interior physical/cyclic disk sector degrees of freedom

$\Gamma=$ partition for physical interface degrees of freedom

Superscripts

b = indicates matrix/vector pertains to blade component

d $=$ indicates matrix/vector pertains to disk component

$h=$ harmonic number

$\mathbf{T}=$ transpose of a matrix or vector

$\mathcal{C B}=$ Craig-Bampton component mode synthesis matrices (also used as a subscript, on transformation matrices)

$\mathcal{S M A}=$ secondary modal analysis matrices

$\sim \quad=$ tilde indicates cyclic coordinates/partitions

- $\quad=$ overbar indicates a vector containing elements (or vectors) repeated once for each sector

\section{Introduction}

B LADED disks, such as those used in turbomachinery, are typically designed with the intent that all blades be identical. In reality, however, there are inevitably small differences among the blades, known as mistuning. Mistuning has been shown to cause vibration behavior that is qualitatively different from that expected of 
a perfectly tuned, ideal bladed disk. The effects of mistuning include localization of free response mode shapes as well as forced response amplitudes and stresses that are much larger than those of the tuned ideal (see [1] for a survey of the recent relevant literature). Failing to account for the effects of mistuning can thus have disastrous effects: accurate prediction of mistuned bladed disk response is essential.

Prediction of mistuned bladed disk response is complicated by the fact that mistuning occurs as essentially random variations due to manufacturing tolerances, material defects, and uneven wear during use. A number of studies have presented reduced-order modeling techniques that generate low-order models of bladed disks from parent finite element models [2-14]. The models generated using these techniques can be run efficiently for large numbers of randomly generated patterns of mistuning, allowing the calculation of the statistics of the maximum forced response amplitudes as a function of amount of mistuning.

However, predicting the response for a specific bladed disk requires knowledge of the particular mistuning pattern present. Traditional bladed disks feature blades that are manufactured individually and attached to the disk by means of a dovetail or fir-tree type connection. These blades can be tested in isolation to determine their deviation from the intended design $[15,16]$. However, many bladed disks are now being designed that feature blades and disk manufactured from a single piece: these bladed disks are known as integrally bladed rotors or blisks. Because the blades of a blisk cannot be easily isolated, identification of mistuning is more difficult. Measurement of the response of individual blades while clamping or damping the disk and adjacent blades is often attempted, yet this can introduce additional mistuning to the system and is not a satisfactory means of isolating blades. To accurately identify mistuning in blisks, a method is needed that uses measurements of the response of the entire structure rather than of individual blades in isolation.

Early work toward this goal was reported by Judge et al. [17-19], in which the authors described a method of using free response system measurements to identify the blade modal stiffnesses present in a reduced-order model of a blisk and described preliminary experimental validation of this technique. Other early work on mistuning identification made use of lumped parameter models [20,21]; more recently, several alternative reduced-order models have been applied to mistuning identification [22-31].

This paper describes in detail two methods for determining the mistuning present in blisks and presents experimental validation of both methods. For both methods, an accurate reduced-order model is developed based on a parent finite element model of a blisk. In the first identification method, measurements of the free response mode shapes of the blisk are used together with the reduced-order model to determine the variations in the modal stiffnesses of the blades. The second identification method is an extension of the first to make use of measurements of the forced response of the blisk. This allows mistuning identification even in cases in which high modal density precludes the isolation of individual system modes.

Section II discusses the formulation of the reduced-order model on which both methods are based. Section III describes the first method, which makes use of free response measurements, whereas the second method, which makes use of forced response measurements, is described in Sec. IV. Both methods are experimentally validated in Sec. $\mathrm{V}$ using a blisk designed specifically to serve as a tuned baseline for studies of mistuning phenomena. Finally, the effectiveness of the methods is discussed and conclusions are drawn in Sec. VI.

\section{Reduced-Order Model}

To identify the mistuning characteristics of individual blades from the dynamic response of an entire bladed disk, two sources of information are used. The first is a theoretical model of the bladed disk, detailed enough that the response could be accurately predicted if all structural parameters were known. The second is a set of measurements of the vibration response of the actual bladed disk, obtained experimentally. If only a few of the parameters in the model are unknown, and the number of measurements equals or exceeds the number of these unknowns, it is then possible to determine the values of the unknown parameters.

A finite element model of a bladed disk typically contains thousands of degrees of freedom (DOF) per sector. Although in reality mistuning originates from small variations in geometry or small material defects, it is convenient to characterize the mistuning of each blade by the degree to which its natural frequencies (or modal stiffnesses) are different from those of the nominal, tuned blade. It is therefore sensible to reduce the finite element model of the bladed disk to one in which blade modal stiffnesses appear directly. Furthermore, it is known that the free vibration modes of the entire bladed disk occur in families, each of which involves blade motion dominated by a particular blade mode shape. Because the small variations that cause mistuning may have a different effect on each blade mode (flexure, torsion, etc.), it is convenient to solve for the mistuning pattern affecting each bladed disk mode family separately. To this end, a reduced-order model is developed in which a single modal stiffness appears explicitly for each blade, corresponding to the dominant blade mode in the bladed disk mode family of interest.

The reduced-order modeling technique developed by Bladh et al. [10] makes use of the Craig-Bampton method of component mode synthesis (CMS) [32] to reduce the size of the finite element model and follows this with a secondary modal analysis (SMA), further reducing model size using a set of modes of the CMS model, to achieve a bladed disk model of minimal size. To perform mistuning identification, the same component mode synthesis method is used, but it is followed by a partial secondary modal analysis, which uses most of the information in the CMS model, but purposefully excludes the dominant blade mode, for which mistuning is to be identified. This modeling approach is similar in spirit to that used by Tan et al. [33] for power flow analysis. The result is a reduced-order model that explicitly retains the blade modal stiffnesses of interest for characterizing mistuning.

\section{A. Component Mode Synthesis}

The disk and each of the $N$ blades are considered substructures of the system. For the Craig-Bampton CMS method [32], two sets of shape vectors are calculated to represent the displacement field for each substructure. The first set, $\boldsymbol{\Phi}$, is a truncated set of the normal vibration mode shapes of the substructure when fixed at the interface with adjacent substructures. The second set, $\boldsymbol{\Psi}$, is a complete set of static "constraint mode" shapes, each resulting from a unit deflection of 1 DOF on the interface, with all other interface DOF held fixed. All shapes in both sets are linearly independent of one another. Furthermore, in the limit that $\boldsymbol{\Phi}$ is not truncated but includes all normal modes of the substructure, the combination of the two sets spans all possible deformations of the substructure, and the CraigBampton method yields exact results relative to the original finite element model.

The mistuning in the bladed disk is considered to be due to variations in the blade substructures only, allowing the modal analysis of the disk substructure to take advantage of cyclic symmetry. Furthermore, the mistuning to be identified is assumed to affect only one vibration mode of the blade substructures: the vibration mode in the frequency range of interest. For each blade, all modal stiffnesses outside the frequency range of interest are considered to be the nominal (tuned) value. In this manner, the final reduced-order model (see Sec. II.B) is of minimal size because only one modal stiffness per blade need be retained explicitly. Thus, the assumption is that, when considering the behavior in a frequency regime where one blade mode is dominant, the mistuning affecting other blade modes can be neglected. This assumption is valid as long as the blade modes are separated significantly in frequency.

\section{Blade Component}

Using the subscript $\Gamma$ to denote DOF on the interface between the blade component and the disk component, and $B$ to indicate interior blade DOF, the physical mass and stiffness matrices of each blade are partitioned as 


$$
\mathbf{K}^{\mathbf{b}}=\left[\begin{array}{ll}
\mathbf{K}_{B B}^{\mathbf{b}} & \mathbf{K}_{B \Gamma}^{\mathbf{b}} \\
\mathbf{K}_{B \Gamma}^{\mathbf{b}^{\mathbf{T}}} & \mathbf{K}_{\Gamma \Gamma}^{\mathbf{b}}
\end{array}\right] \quad \mathbf{M}^{\mathbf{b}}=\left[\begin{array}{ll}
\mathbf{M}_{B B}^{\mathbf{b}} & \mathbf{M}_{B \Gamma}^{\mathbf{b}} \\
\mathbf{M}_{B \Gamma}^{\mathbf{b}^{\mathbf{T}}} & \mathbf{M}_{\Gamma \Gamma}^{\mathbf{b}}
\end{array}\right]
$$

The normal mode shapes of the blade fixed at the blade-disk interface $\phi_{\mathbf{b}}$ and the associated modal stiffnesses $\lambda_{b}$ are found by solving the conventional eigenvalue problem on the interior blade DOF:

$$
\left[\mathbf{K}_{B B}^{\mathbf{b}}-\lambda_{b} \mathbf{M}_{B B}^{\mathbf{b}}\right] \phi_{b}=\mathbf{0}
$$

A subset of the normal mode shapes are retained and assembled into the matrix $\boldsymbol{\Phi}_{b}$, which is partitioned as $\left[\begin{array}{ll}\phi_{b_{m}} & \boldsymbol{\Phi}_{b_{t}}\end{array}\right]$ to separate the single mode shape corresponding to the blade mode for which mistuning is to be identified $\phi_{b_{m}}$ from the remaining retained shapes, which are assumed to be unaffected by mistuning. The corresponding eigenvalue is denoted $\lambda_{b_{m}}$, and the mistuning to be identified is considered to be variations in $\lambda_{b_{m}}$ such that the modal stiffness of an actual, mistuned blade $\lambda_{\text {mist }_{n}}$ is $\left(1+\delta_{n}\right) \lambda_{b_{m}}$, where $n$ is the index of the blade in question.

The constraint mode shapes of the blade substructure are found by considering the static problem

$$
\left[\begin{array}{ll}
\mathbf{K}_{B B} & \mathbf{K}_{B \Gamma} \\
\mathbf{K}_{B \Gamma}^{\mathbf{T}} & \mathbf{K}_{\Gamma \Gamma}
\end{array}\right]\left[\begin{array}{c}
\mathbf{\Psi}_{b} \\
\mathbf{I}
\end{array}\right]=\left[\begin{array}{c}
\mathbf{0} \\
\mathbf{R}_{\Gamma}
\end{array}\right]
$$

where $\mathbf{R}_{\Gamma}$ is a vector of reaction forces at the interface DOF due to the imposed constraints. Only the top portion of Eq. (3) needs to be considered to solve for the constraint mode shapes: $\Psi_{b}=$ $-\mathbf{K}_{B B}^{-1} \mathbf{K}_{B \Gamma}$.

The matrix $\boldsymbol{\Psi}_{b}$ of blade constraint modes, all of which are retained, is used together with the retained blade normal mode shapes to reduce the physical coordinates of the blade interior $\mathbf{x}_{B}^{\mathbf{b}}$ to a smaller set of blade modal coordinates, which includes a single coordinate $p_{b_{m}}^{b}$, representing the amplitude of the dominant blade mode (for which mistuning is to be identified), and a vector of coordinates $\mathbf{p}_{b_{t}}^{\mathbf{b}}$ representing the amplitudes of the other retained blade normal modes. Meanwhile, the physical coordinates on the disk-blade interface $\mathbf{x}_{\Gamma}^{\mathbf{b}}$ are retained as constraint mode coordinates $\mathbf{p}_{c}^{\mathbf{b}}$ :

$$
\mathbf{x}^{\mathbf{b}}=\left\{\begin{array}{c}
\mathbf{x}_{B}^{\mathbf{b}} \\
\mathbf{x}_{\Gamma}^{\mathbf{b}}
\end{array}\right\}=\left[\begin{array}{ccc}
\phi_{b_{m}} & \boldsymbol{\Phi}_{b_{t}} & \boldsymbol{\Psi}_{b} \\
\mathbf{0} & \mathbf{0} & \mathbf{I}
\end{array}\right]\left\{\begin{array}{c}
p_{b_{m}}^{b} \\
\mathbf{p}_{b_{t}}^{\mathbf{b}} \\
\mathbf{p}_{c}^{\mathbf{b}}
\end{array}\right\}=\mathbf{U}_{\mathcal{C B}}^{\mathbf{b}} \mathbf{p}^{\mathbf{b}}
$$

The Craig-Bampton $\mathcal{C B}$ modal matrix that converts from physical to blade modal coordinates can be used to obtain the reduced mass and stiffness matrices of the nominal blade:

$$
\begin{gathered}
\boldsymbol{\mu}^{\mathbf{b}}=\mathbf{U}_{\mathcal{C B}}^{\mathbf{b}^{\mathbf{T}}} \mathbf{M}^{\mathbf{b}} \mathbf{U}_{\mathcal{C B}}^{\mathbf{b}}=\left[\begin{array}{ccc}
\mathbf{1} & \mathbf{0} & \boldsymbol{\mu}_{b_{m} c} \\
\mathbf{0} & \mathbf{I} & \boldsymbol{\mu}_{b_{t} c} \\
\boldsymbol{\mu}_{b_{m} c}^{\mathbf{T}} & \boldsymbol{\mu}_{b_{t} c}^{\mathbf{T}} & \boldsymbol{\mu}_{c c, b}
\end{array}\right] \\
\boldsymbol{\kappa}^{\mathbf{b}}=\mathbf{U}_{\mathcal{C B}}^{\mathbf{b}^{\mathbf{T}}} \mathbf{K}^{\mathbf{b}} \mathbf{U}_{\mathcal{C B}}^{\mathbf{b}}=\left[\begin{array}{ccc}
\lambda_{b_{m}} & \mathbf{0} & \mathbf{0} \\
\mathbf{0} & \boldsymbol{\Lambda}_{b_{t}} & \mathbf{0} \\
\mathbf{0} & \mathbf{0} & \boldsymbol{\kappa}_{c c, b}
\end{array}\right]
\end{gathered}
$$

Here, $\boldsymbol{\Lambda}_{b_{t}}$ is a diagonal matrix consisting of the eigenvectors $\lambda_{b}$ corresponding to the normal modes retained in $\boldsymbol{\Phi}_{b_{t}}$. The inclusion of the blade constraint modes $\boldsymbol{\Psi}_{b}$ results in off-diagonal terms in the mass and stiffness matrices $\boldsymbol{\mu}^{\mathbf{b}}$ and $\boldsymbol{\kappa}^{\mathbf{b}}$ : the partition subscripts $b_{m} c$ and $b_{t} c$ refer to terms coupling the constraint mode motion to mistuned and tuned blade normal mode motion, respectively, whereas the subscript $c c, b$ refers to the partitions that include crosscoupling between blade constraint modes. Note that $\phi_{b_{m}}$ and $\boldsymbol{\Phi}_{b_{t}}$ have been normalized such that the modal masses have unit value.

\section{Disk Component}

The analysis of the disk component follows that presented by Bladh et al. [10], summarized briefly here. $N$ copies of the physical coordinates $\mathbf{x}^{\mathrm{d}}$ for each disk sector are assembled into a vector $\overline{\mathbf{x}}^{\mathbf{d}}$ for the entire disk. Before computing the normal modes $\boldsymbol{\Phi}_{d}$ and the constraint modes $\boldsymbol{\Psi}_{d}$ of the disk substructure, these physical coordinates $\overline{\mathbf{x}}^{\mathbf{d}}$ are first transformed to cyclic coordinates $\tilde{\mathbf{u}}$, so that motion of the disk in all $N$ sectors can be represented by motion in a single sector for $N$ harmonics. This transformation is accomplished using the real-valued Fourier matrix $\mathbf{F}$ (see [10] or [34]):

$$
\overline{\mathbf{x}}^{\mathbf{d}}=\hat{\mathbf{F}} \tilde{\mathbf{u}}
$$

where $\hat{\mathbf{F}}=(\mathbf{F} \otimes \mathbf{I})$, that is, the Fourier matrix is expanded by means of the Kronecker product with an identity matrix $\mathbf{I}$ whose size is the number of coordinates in each sector. In this way, $\hat{\mathbf{F}}$ transforms $N$ vectors of coordinates rather than $N$ individual coordinates.

The stiffness matrix for a single disk sector is denoted $\mathbf{K}^{\mathbf{d}}$ and is similar to the blade stiffness matrix in Eq. (1), expect with partitions for the blade-disk interface $\Gamma$ and disk sector $\mathbf{S}$ degrees of freedom. The expanded stiffness matrix for all harmonics is the same as the stiffness matrix for all sectors, that is, $\tilde{\mathbf{K}}^{\mathbf{d}}=\hat{\mathbf{F}}^{\mathbf{T}}\left(\mathbf{I} \otimes \mathbf{K}^{\mathbf{d}}\right) \hat{\mathbf{F}}=$ $\left(\mathbf{I} \otimes \mathbf{K}^{\mathbf{d}}\right)$. However, in physical coordinates, connectivity between adjacent sectors creates a set of constraint equations that couple all blocks of $\mathbf{I} \otimes \mathbf{K}^{\mathbf{d}}$ to one another, whereas in cyclic coordinates, the various harmonics are decoupled from one another, and the solution of the cyclic eigenvalue problem can be found one harmonic at a time using

$$
\left[\tilde{\mathbf{K}}_{S S}^{\mathbf{d}, h}-\lambda_{d} \tilde{\mathbf{M}}_{S S}^{\mathbf{d}, h}\right] \tilde{\phi}_{d}^{h}=\mathbf{0}, \quad h=0, \ldots, P
$$

where $h$ indicates the harmonic number and $P=\operatorname{int}[N / 2]$, and where the subscript $S S$ refers to the portions of the cyclic disk matrices pertaining to interactions among interior disk sector degrees of freedom. Calculation of the cyclic constraint modes via $\tilde{\boldsymbol{\Psi}}_{d}^{h}=$ $-\tilde{\mathbf{K}}_{S S}^{\mathbf{d}, h^{-1}} \tilde{\mathbf{K}}_{S \Gamma}^{\mathbf{d}, h}$ also involves the partition of the cyclic disk stiffness matrix that couples interior sector $S$ DOF to disk-blade interface $\Gamma$ DOF.

The retained cyclic normal mode shapes are assembled into a matrix $\tilde{\boldsymbol{\Phi}}_{d}^{h}$ and used together with the complete matrix of cyclic constraint mode shapes $\tilde{\boldsymbol{\Psi}}_{d}^{h}$ to reduce the cyclic physical disk coordinates $\tilde{\mathbf{u}}_{S}^{h}$ to cyclic disk modal coordinates $\tilde{\mathbf{p}}_{d}^{\mathbf{d}^{h}}$. The cyclic physical blade-disk interface coordinates $\tilde{\mathbf{u}}_{\Gamma}^{h}$ are retained as cyclic constraint modal coordinates $\tilde{\mathbf{p}}_{c}^{\mathbf{d}^{h}}$. These transformations are assembled into a cyclic Craig-Bampton modal matrix $\tilde{\mathbf{U}}_{\mathcal{C B}}^{\mathbf{d}}$, so that the transformation for all coordinates is

$$
\begin{aligned}
& \tilde{\mathbf{u}}=\tilde{\mathbf{U}}_{\mathcal{C B}}^{\mathbf{d}} \tilde{\mathbf{p}}^{\mathbf{d}}=\left\{\begin{array}{c}
\tilde{\mathbf{u}}_{\mathbf{D}} \\
\tilde{\mathbf{u}}_{\Gamma}
\end{array}\right\} \\
& =\left[\begin{array}{cc}
\tilde{\mathbf{B}} \operatorname{diag}_{h=0, \ldots, P}\left[\tilde{\boldsymbol{\Phi}}_{d}^{h}\right] & \tilde{\mathbf{B}} \operatorname{diag}_{h=0, \ldots, P}\left[\tilde{\mathbf{\Psi}}_{d}^{h}\right] \\
\mathbf{0} & \mathbf{I}
\end{array}\right]\left\{\begin{array}{c}
\tilde{\mathbf{p}}_{d}^{\mathbf{d}} \\
\tilde{\mathbf{p}}_{c}^{\mathbf{d}}
\end{array}\right\}
\end{aligned}
$$

with the matrices $\tilde{\boldsymbol{\Phi}}_{d}^{h}$ and $\tilde{\boldsymbol{\Psi}}_{d}^{h}$ occurring as blocks (for each harmonic $h$ ) along the diagonal of their respective partitions. $\tilde{\mathbf{B}} \mathbf{d i a g}[\cdot]$ is used to denote a pseudo-block-diagonal matrix, which is similar to a block-diagonal matrix except that the blocks on the diagonal are not all the same size. This is because of coupling between "cosine" and "sine" versions of the same harmonic, which exist for all harmonics except $h=0$, and, if $N$ is even, $h=P$.

Assembling all harmonics of the cyclic disk mass and stiffness matrices and applying the transformation in Eq. (8) yields the reduced mass and stiffness matrices for the disk component:

$$
\tilde{\boldsymbol{\mu}}^{\mathbf{d}}=\left[\begin{array}{cc}
\mathbf{I} & \tilde{\boldsymbol{\mu}}_{d c} \\
\tilde{\boldsymbol{\mu}}_{d c}^{\mathbf{T}} & \tilde{\boldsymbol{\mu}}_{c c, d}
\end{array}\right] \quad \tilde{\boldsymbol{\kappa}}^{\mathbf{d}}=\left[\begin{array}{cc}
\tilde{\boldsymbol{\Lambda}}_{d} & \mathbf{0} \\
\mathbf{0} & \tilde{\boldsymbol{\kappa}}_{c c, d}
\end{array}\right]
$$

As in the case of the blade components, $\tilde{\boldsymbol{\Lambda}}_{d}$ is a diagonal matrix consisting of the retained disk eigenvalues, and $\tilde{\boldsymbol{\mu}}^{\mathbf{d}}$ and $\tilde{\boldsymbol{\kappa}}^{\mathbf{d}}$ contain off-diagonal terms; in this case, they represent interaction between cyclic disk normal modes $d$ and constraint modes $c$ or among the constraint modes themselves (within the $c c, d$ partition). 


\section{Component Mode Synthesis Model Assembly}

Once the size of each substructure model is reduced by retaining a limited set of normal modes, the substructures are reassembled by enforcing displacement compatibility on the component interfaces, $\overline{\mathbf{p}}_{c}^{\mathbf{b}}=\hat{\mathbf{F}} \tilde{\mathbf{p}}_{c}^{\mathbf{d}}$, where the overbar indicates a vector composed of $N$ sets of the vector $\mathbf{p}_{c}^{\mathbf{b}}$, one for each blade. The Fourier matrix is necessary to match the interface DOF on the blade components, on each of $N$ blades, with the cyclic interface DOF on the disk component (for each of $N$ harmonics):

$$
\left\{\begin{array}{c}
\tilde{\mathbf{p}}_{d}^{\mathbf{d}} \\
\tilde{\mathbf{p}}_{c}^{\mathbf{d}} \\
\overline{\mathbf{p}}_{b_{m}}^{\mathbf{b}} \\
\overline{\mathbf{p}}_{b_{t}}^{b_{t}} \\
\overline{\mathbf{p}}_{c}^{\mathbf{b}}
\end{array}\right\}=\left[\begin{array}{cccc}
\mathbf{I} & \mathbf{0} & \mathbf{0} & \mathbf{0} \\
\mathbf{0} & \mathbf{I} & \mathbf{0} & \mathbf{0} \\
\mathbf{0} & \mathbf{0} & \mathbf{0} & \mathbf{I} \\
\mathbf{0} & \mathbf{0} & \hat{\mathbf{F}} & \mathbf{0} \\
\mathbf{0} & \hat{\mathbf{F}} & \mathbf{0} & \mathbf{0}
\end{array}\right]\left\{\begin{array}{c}
\tilde{\mathbf{p}}_{d} \\
\tilde{\mathbf{p}}_{c} \\
\tilde{\mathbf{p}}_{b_{t}} \\
\overline{\mathbf{p}}_{b_{m}}
\end{array}\right\}=\mathbf{T}_{\mathcal{C B}} \mathbf{p}
$$

Note that the tuned blade modal coordinates have also been transformed to cyclic coordinates: $\tilde{\mathbf{p}}_{d}, \tilde{\mathbf{p}}_{c}$, and $\tilde{\mathbf{p}}_{b_{t}}$ are cyclic modal coordinates corresponding to disk, constraint, and tuned blade mode vibration, respectively. The only noncyclic coordinates remaining are the mistuned blade modal coordinates $\overline{\mathbf{p}}_{b_{m}}$, of which there is one per blade.

The transformation $\mathbf{T}_{\mathcal{C B}}$ is used to create synthesized CraigBampton matrices. The stiffness matrix is

$$
\mathbf{K}^{\mathcal{C B}}=\mathbf{T}_{\mathcal{C B}}^{\mathbf{T}}\left[\begin{array}{cc}
\tilde{\boldsymbol{\kappa}}^{\mathbf{d}} & \mathbf{0} \\
\mathbf{0} & \overline{\boldsymbol{\kappa}}^{\mathbf{b}}
\end{array}\right] \mathbf{T}_{\mathcal{C B}}
$$

and the mass matrix is analogous.

\section{B. Secondary Modal Analysis on Disk, Interface, and Tuned Blade Modes}

The Craig-Bampton stiffness matrix $\mathbf{K}^{\mathcal{C B}}$ explicitly contains, for each blade, the modal stiffnesses corresponding to the blade mode for which mistuning is to be identified, $\boldsymbol{\Lambda}_{b_{m}}=\lambda_{b_{m}} \mathbf{I}$. It also contains the disk normal modes, remaining (tuned) blade normal modes, and constraint modes coupling disk to blade behavior, all in cyclic coordinates (one per harmonic). The latter can be grouped into a single " $e$ " partition such that

$$
\mathbf{K}^{\mathcal{C B}}=\left[\begin{array}{cc}
\mathbf{K}_{e}^{\mathcal{C B}} & \mathbf{0} \\
\mathbf{0} & \boldsymbol{\Lambda}_{b_{m}}
\end{array}\right]
$$

Likewise,

$$
\mathbf{M}^{\mathcal{C B}}=\left[\begin{array}{cc}
\mathbf{M}_{e}^{\mathcal{C B}} & \mathbf{M}_{e b_{m}}^{\mathcal{C B}} \\
\mathbf{M}_{e b_{m}}^{\mathcal{C B}} & \mathbf{I}
\end{array}\right]
$$

Because the $\mathbf{K}_{e}^{\mathcal{C B}}$ and $\mathbf{M}_{e}^{\mathcal{C B}}$ partitions are represented entirely in cyclic coordinates and contain no interharmonic coupling, a secondary modal analysis can be carried out one harmonic at a time by solving

$$
\left[\tilde{\mathbf{K}}_{e}^{h}-\lambda_{e}^{h} \tilde{\mathbf{M}}_{e}^{h}\right] \tilde{\boldsymbol{\theta}}_{e}^{h}=\mathbf{0}, \quad h=0, \ldots, P
$$

where $\tilde{\mathbf{K}}_{e}^{h}$ and $\tilde{\mathbf{M}}_{e}^{h}$ are the portions of $\mathbf{K}_{e}^{\mathcal{C B}}$ and $\mathbf{M}_{e}^{\mathcal{C B}}$ corresponding to harmonic $h$.

The modes $\tilde{\boldsymbol{\theta}}_{e}$ represent the combined effects of the disk, constraint, and tuned blade mode motion, that is, all effects except the motion in the fixed-interface blade normal mode for which mistuning is to be identified. A subset of these "secondary" modes is selected for each harmonic, and the individual partitions of $\tilde{\boldsymbol{\theta}}_{e}^{h}\left(\tilde{\boldsymbol{\theta}}_{d}^{h}, \tilde{\boldsymbol{\theta}}_{c}^{h}\right.$, and $\left.\tilde{\boldsymbol{\theta}}_{b_{t}}^{h}\right)$ are assembled for all harmonics into the matrices $\tilde{\boldsymbol{\Theta}}_{d}, \tilde{\boldsymbol{\Theta}}_{c}$, and $\tilde{\boldsymbol{\Theta}}_{b_{t}}$. The transformation from CMS coordinates $\mathbf{p}$ to secondary modal analysis $\mathcal{S M} \mathcal{A}$ coordinates $\mathbf{q}$ is

$$
\mathbf{p}=\left\{\begin{array}{c}
\tilde{\mathbf{p}}_{d} \\
\tilde{\mathbf{p}}_{c} \\
\tilde{\mathbf{p}}_{b_{t}} \\
\overline{\mathbf{p}}_{b_{m}}
\end{array}\right\}=\left[\begin{array}{cc}
\tilde{\boldsymbol{\Theta}}_{d} & \mathbf{0} \\
\tilde{\boldsymbol{\Theta}}_{c} & \mathbf{0} \\
\tilde{\boldsymbol{\Theta}}_{b_{t}} & \mathbf{0} \\
\mathbf{0} & \mathbf{I}
\end{array}\right]\left\{\begin{array}{c}
\tilde{\mathbf{q}}_{e} \\
\overline{\mathbf{q}}_{m}
\end{array}\right\}=\mathbf{U}^{\mathcal{S M A}} \mathbf{q}
$$

The SMA coordinates are divided into an " $m$ " partition containing one mistuned blade modal coordinate per blade, and the cyclic $e$ partition, containing, for each harmonic, coordinates for each mode of the secondary modal analysis. The transformation is applied to the CMS mass and stiffness matrices to achieve

$$
\begin{gathered}
\mathbf{M}^{\mathcal{S M A}}=\left[\begin{array}{cc}
\mathbf{I} & \mathbf{M}_{e m}^{\mathcal{S M} \mathcal{A}^{\mathrm{T}}} \\
\mathbf{M}_{e m}^{\mathcal{S M A}} & \mathbf{I}
\end{array}\right] \\
\mathbf{K}^{\mathcal{S M A}}=\left[\begin{array}{cc}
\tilde{\boldsymbol{\Lambda}}_{e}^{\mathcal{S M A}} & \mathbf{0} \\
\mathbf{0} & \boldsymbol{\Lambda}_{b_{m}}
\end{array}\right]
\end{gathered}
$$

where $\mathbf{M}_{e m}^{\mathcal{S M A}}=\left(\mathbf{I} \otimes \boldsymbol{\mu}_{b_{m}}\right) \hat{\mathbf{F}} \tilde{\mathbf{\Theta}}_{c}^{\mathcal{S M A}}$. The cyclic modal stiffness matrix $\tilde{\Lambda}_{e}^{\mathcal{S M A}}$ contains the eigenvalues corresponding to the subset of the secondary modes $\tilde{\boldsymbol{\theta}}_{e}$ that are retained. The blade modal stiffness matrix $\boldsymbol{\Lambda}_{b_{m}}$ is merely $\lambda_{b_{m}} \mathbf{I}$ in the case of a perfectly tuned system; each element is replaced by $\lambda_{\text {mist }_{n}}=\left(1+\delta_{n}\right) \lambda_{b_{m}}$ for blade $n$ from 1 to $N$ to represent mistuning in the model.

The smallest model possible is one in which only one secondary mode $\tilde{\boldsymbol{\theta}}_{e}^{h}$ is retained per harmonic: the matrix size is then $2 N(N$ is both the number of blades and the number of harmonics). Retaining more secondary modes per harmonic increases accuracy but still provides a model of modest size, $N\left(N_{e}+1\right)$, where $N_{e}$ is the number of secondary modes retained per harmonic. Note that the accuracy of the final model is affected by the number of modes retained in both the CMS and SMA reductions, but the size is determined solely by the SMA reduction. Retaining as many modes as feasible in the CMS reduction thus provides a highly accurate final model without sacrificing small model size.

\section{Identification Method Using Free Response Measurements}

For bladed disks with very low damping, distinct resonant peaks can be measured experimentally that correspond to vibration dominated by a single mode. By determining the natural frequencies and mode shapes of the system, the mistuning parameters of individual blades can be identified using the reduced-order model. The undamped free vibration eigenvalue problem for the reducedorder model [see Eqs. (15-17)] is

$$
\left[-\omega^{2} \mathbf{M}^{\mathcal{S M A}}+\mathbf{K}^{\mathcal{S M A}}\right]\{\mathbf{q}\}=\left[\begin{array}{cc}
\mathbf{A}_{e} & \mathbf{A}_{e m}^{\mathbf{T}} \\
\mathbf{A}_{e m} & \mathbf{A}_{m}
\end{array}\right]\left\{\begin{array}{c}
\tilde{\mathbf{q}}_{e} \\
\overline{\mathbf{q}}_{m}
\end{array}\right\}=0
$$

Here, the matrix $\mathbf{A}$ is partitioned in the same manner as $\mathbf{M}^{\mathcal{S M A}}$ and $\mathbf{K}^{\mathcal{S M A}}$ in Eqs. (16) and (17). All unknown mistuning parameters occur in the $\mathbf{A}_{m}$ partition, a diagonal matrix whose elements are $-\omega^{2}+\lambda_{\text {mist }_{n}}$. Each system eigenvalue $\omega^{2}$ is the square of a measured frequency, but the corresponding eigenvector $\mathbf{q}$ must be determined from measurements of physical coordinates. A combination of the transformations in Eqs. (4), (10), and (15) yields the relation between physical blade coordinates $\overline{\mathbf{x}_{B}^{\mathrm{b}}}$ and the SMA coordinates $\mathbf{q}$ :

$$
\overline{\mathbf{x}}_{B}^{\mathbf{b}}=[\mathbf{V}]\left\{\begin{array}{l}
\tilde{\mathbf{q}}_{e} \\
\overline{\mathbf{q}}_{m}
\end{array}\right\}
$$

where

$$
\mathbf{V}=\left\{\left[\left(\mathbf{I} \otimes \boldsymbol{\Phi}_{b_{t}}\right) \hat{\mathbf{F}}\left[\tilde{\boldsymbol{\Theta}}_{b_{t}}\right]+\left(\mathbf{I} \otimes \boldsymbol{\Psi}_{b}\right) \hat{\mathbf{F}}\left[\tilde{\boldsymbol{\Theta}}_{c}\right]\right] \quad \mathbf{I} \otimes \phi \mathbf{b}_{m}\right\}
$$

If every DOF in the original finite element model could be measured, $\mathbf{q}$ could be found by inverting $\mathbf{V}$.

Fortunately, each mode shape can be determined using a much smaller set of measurements. Removing the $N$ rows of Eq. (18) that contain the $\mathbf{A}_{m}$ partition leaves $N_{e} N$ equations for the $N\left(\overline{N_{e}}+1\right)$ 
DOF in $\mathbf{q}$. By making one measurement per blade and extracting the corresponding $N$ rows from $\mathbf{V}$,

$$
\overline{\mathbf{x}}_{\text {meas }}^{\mathbf{b}}=\left[\mathbf{V}_{\text {meas }}\right]\left\{\begin{array}{l}
\tilde{\mathbf{q}}_{e} \\
\overline{\mathbf{q}}_{m}
\end{array}\right\}
$$

$N$ additional equations are obtained that relate $\mathbf{q}$ to the measured physical vibration amplitudes.

These $N$ equations replace the bottom partitions of Eq. (18), and the result is solved for the SMA mode shape $\mathbf{q}$

$$
\mathbf{q}=\left\{\begin{array}{c}
\tilde{\mathbf{q}}_{e} \\
\overline{\mathbf{q}}_{m}
\end{array}\right\}=\left[\begin{array}{cc}
\mathbf{A}_{e} & \mathbf{A}_{e m}^{\mathbf{T}} \\
& \mathbf{V}_{\text {meas }}
\end{array}\right]^{-1}\left\{\begin{array}{c}
0 \\
\overline{\mathbf{x}}_{\text {meas }}^{\mathbf{b}}
\end{array}\right\}
$$

Once $\mathbf{q}$ is known, the bottom partitions of Eq. (18) (which consist of $N$ equations) can now be solved for the unknown mistuning contained in $\mathbf{A}_{m}$ :

$$
\begin{aligned}
& {\left[\begin{array}{ll}
\mathbf{A}_{e m} & \mathbf{A}_{m}
\end{array}\right]\left\{\begin{array}{c}
\tilde{\mathbf{q}}_{e} \\
\overline{\mathbf{q}}_{m}
\end{array}\right\}} \\
& \quad=\left[-\omega^{2} \mathbf{M}_{e m}^{\mathcal{S M A}}-\omega^{2} \mathbf{I}+\operatorname{diag}_{n=1, \ldots, N} \lambda_{\text {mist }_{n}}\right]\left\{\begin{array}{c}
\tilde{\mathbf{q}}_{e} \\
\overline{\mathbf{q}}_{m}
\end{array}\right\}=\mathbf{0}
\end{aligned}
$$

Here, $\operatorname{diag}[\cdot]$ indicates a diagonal matrix, such that

$$
\operatorname{diag}_{n=1, \ldots, N} \lambda_{\text {mist }_{n}}
$$

is an $n \times n$ matrix with the individual blades' mistuned modal stiffnesses along the diagonal. Each such stiffness (the square of the mistuned frequency) is thus

$$
\lambda_{\text {mist }_{n}}=\omega^{2}\left(1-\frac{\left(\mathbf{M}_{e m}^{\mathcal{S M A}} \tilde{\mathbf{q}}_{e}\right)_{n}}{q_{m_{n}}}\right), \quad n=1, \ldots, N
$$

where $\left(\mathbf{M}_{e m}^{\mathcal{S M} \mathcal{A}} \tilde{\mathbf{q}}_{e}\right)_{n}$ refers to the $n$th row of $\mathbf{M}_{e m}^{\mathcal{S M A}}$ multiplied by $\tilde{\mathbf{q}}_{e}$, and $q_{m_{n}}$ is the $n$th component of $\overline{\mathbf{q}}_{m}$. Finally, since $\lambda_{\text {mist }_{n}}=$ $\left(1+\delta_{n}\right) \lambda_{b_{m}}$, each mistuned blade eigenvalue can in turn be solved for $\delta_{n}$, the individual blade mistuning:

$$
\delta_{n}=\frac{\lambda_{\text {mist }_{n}}}{\lambda_{b_{m}}}-1 \quad n=1, \ldots, N
$$

Equations (21), (23), and (24) precisely predict blade mistuning $\delta_{n}$ only when $\mathbf{q}$ is an exact mode shape of the SMA model and $\omega$ is the corresponding natural frequency. Imprecision in measurement, discrepancies between the physical system and the finite element model, and any inaccuracies introduced in generating the reducedorder model all create differences between the actual measured data $\overline{\mathbf{x}}_{\text {meas }}^{\mathbf{b}}$ and the "perfect" data necessary for an exact calculation of $\mathbf{q}$ and thus exact identification of the mistuning. To examine the sensitivity of the process to measurement error, the mistuning parameters are calculated for a large number of cases in which the measurements are perturbed by a random amount (with a uniform distribution), and the standard deviation of the resulting identifications is compared with the standard deviation of the measurement perturbations. The ratio is termed $\beta$, the error magnification factor, and is calculated separately for each blade. If $\beta_{n}$ is large, the error in the mistuning identification for blade $n$ will be greater than the inaccuracy in the measured data. If $\beta_{n}$ is small, the calculation of $\delta_{n}$ is insensitive to variations in the measured data, and thus mistuning for that blade can be known with greater confidence.

Examination of Eqs. (21) and (23) gives some insight into circumstances giving rise to particularly high or low values of $\beta$. First, the ratio $\left(\mathbf{M}_{e m}^{\mathcal{S M} \mathcal{A}} \tilde{\mathbf{q}}_{e}\right)_{n} / q_{m_{n}}$ is least sensitive to errors in $\mathbf{q}$ when the denominator is large, that is, for blades with high amplitude. Second, the calculation of $\lambda_{\text {mist }_{n}}$ is least sensitive to errors in $\left(\mathbf{M}_{e m}^{\mathcal{S M} \mathcal{A}} \tilde{\mathbf{q}}_{e}\right)_{n} / q_{m_{n}}$ when that ratio is close to zero, so that $\omega^{2} \approx \lambda_{\text {mist }_{n}}$, that is, when the natural frequency of the measured system mode is near the blade-alone natural frequency. These two observations can be combined to form a pseudoanalytical approximation for $\beta$ :

$$
\beta \approx\left[\left(\frac{x_{\max }}{x_{n}}\right)\left(1-\frac{\omega^{2}}{\lambda_{b_{n}}}\right)\right]
$$

where $x_{n}$ is the measurement on the blade in question, $x_{\max }$ is the measurement on the blade with maximum amplitude, $\omega$ is the measured frequency of the system mode, and $\lambda_{b_{n}}$ is the modal stiffness of the blade in question.

Because different system modes feature large amplitude response in different blades, especially in the presence of localization, a composite of results from several system modes may be necessary to give high confidence in the mistuning identification for every blade. For each blade $n$, mistuning $\delta_{n}$ and error magnification $\beta_{n}$ are calculated several times, once for each of several measurements. Every $\delta_{n}$ with corresponding $\beta_{n}$ above a certain tolerance is eliminated, and the remaining identified mistuning parameters are averaged to provide a single result for each blade. The tolerance should be adjusted to provide the most accurate identification while still providing at least one valid $\delta$ per blade.

\section{Identification Method Using Forced Response Measurements}

The identification technique can be extended to use measurements of forced response rather than of a free response mode shape. For harmonic motion, the forced response equivalent of Eq. (18) is

$$
\begin{gathered}
{\left[-\omega^{2} \mathbf{M}^{\mathcal{S M A}}+i \omega \mathbf{C}^{\mathcal{S M A}}+(1+i G) \mathbf{K}^{\mathcal{S M A}}\right]\{\mathbf{q}\}} \\
\quad=\left[\begin{array}{cc}
\mathbf{A}_{e} & \mathbf{A}_{e m}^{\mathbf{T}} \\
\mathbf{A}_{e m} & \mathbf{A}_{m}
\end{array}\right]\left\{\begin{array}{c}
\tilde{\mathbf{q}}_{e} \\
\overline{\mathbf{q}}_{m}
\end{array}\right\}=\mathbf{F}^{\mathcal{S M A}}
\end{gathered}
$$

where $i$ denotes $\sqrt{-1}, \mathbf{C}^{\mathcal{S M A}}$ is a matrix accounting for viscous damping, $G$ is a structural damping coefficient, and $\mathbf{F}^{\mathcal{S M A}}$ is any forcing vector representing harmonic forcing at frequency $\omega$. Note that $\mathbf{q}$ is no longer an eigenvector; it is now the forced response vibration pattern in SMA coordinates at the frequency $\omega$.

If the forcing is known precisely, the differences from the free response technique are minimal: $\mathbf{q}$ is determined in the same manner, and Eq. (22) is merely modified to include the effects of damping on the left side and forcing on the right. If viscous damping is neglected, the resulting equations can be solved directly for $\lambda_{\text {mist }}$; if viscous damping is included, they become quadratic equations in $\sqrt{\lambda_{\text {mist }}}$.

However, if the forcing is not completely known, mistuning can still be identified. The specimen, which has unknown mistuning $\delta$ (consisting of $\delta_{n}$ for each blade, $n=1, \ldots, N$ ), is subject to a temporally harmonic forcing at a known frequency, but with a spatial distribution and amplitude that need not be known. Measurements are taken of the vibration amplitude and phase at one location on each blade, exactly as in the free response method. The specimen is then mistuned additionally by a known amount $\boldsymbol{\Delta} \boldsymbol{\delta}$, consisting of one $\Delta \delta_{n}$ for each blade, $n=1, \ldots, N$. Note that all blades need not be altered, that is, any $\boldsymbol{\Delta} \boldsymbol{\delta}$ can be used that has at least one nonzero $\Delta \delta_{n}$. Measurements are taken a second time with the forcing exactly the same as before. A third set of measurements is taken after adding an additional scalar multiple of the added mistuning $\alpha \boldsymbol{\Delta} \boldsymbol{\delta}$ (or, equivalently, after removing the added mistuning $\Delta \boldsymbol{\delta}$ and replacing it with $(1+\alpha) \boldsymbol{\Delta} \boldsymbol{\delta})$.

The input to the mistuning identification algorithm is now three sets of measurements in physical coordinates $\overline{\mathbf{x}}_{\text {meas }_{1}}^{\mathbf{b}}, \overline{\mathbf{x}}_{\text {meas }_{2}}^{\mathbf{b}}$, and $\overline{\mathbf{x}}_{\text {meas }}^{\mathbf{b}}$ corresponding to three different mistuning patterns $\boldsymbol{\delta}_{1}, \boldsymbol{\delta}_{2}$, and $\boldsymbol{\delta}_{3}$, all of which are unknown, but which differ from each other by known amounts, such that $\boldsymbol{\delta}_{2}=\boldsymbol{\delta}_{1}+\boldsymbol{\Delta} \boldsymbol{\delta}$ and $\boldsymbol{\delta}_{3}=\boldsymbol{\delta}_{2}+\alpha \boldsymbol{\Delta} \boldsymbol{\delta}$. The corresponding forced response problems [three versions of Eq. (26)] will differ only in the mistuned blade partition $\mathbf{A}_{m}$ and the response $\mathbf{q}$; the forcing vector $\mathbf{F}^{\mathcal{S M A}}$ and all other partitions of the $\mathbf{A}$ matrix are the same for all three cases. By subtracting one case from another, the forcing information can be eliminated: 


$$
\begin{aligned}
& {\left[\begin{array}{cc}
\mathbf{A}_{e} & \mathbf{A}_{e m}^{\mathbf{T}} \\
\mathbf{A}_{e m} & \mathbf{A}_{m}^{\text {tuned }}
\end{array}\right]\left\{\boldsymbol{\Delta} \mathbf{q}_{j k}\right\}+\left[\begin{array}{cc}
\mathbf{0} & \mathbf{0} \\
\mathbf{0} & \boldsymbol{\Delta} \mathbf{A}_{j}
\end{array}\right]\left\{\mathbf{q}_{j}\right\}-\left[\begin{array}{cc}
\mathbf{0} & \mathbf{0} \\
\mathbf{0} & \boldsymbol{\Delta} \mathbf{A}_{k}
\end{array}\right]\left\{\mathbf{q}_{k}\right\}} \\
& =\left\{\begin{array}{l}
\mathbf{0} \\
\mathbf{0}
\end{array}\right\} \quad(j, k)=(1,2),(2,3)
\end{aligned}
$$

Here, the matrices $\boldsymbol{\Delta} \mathbf{A}_{j}$ and $\boldsymbol{\Delta} \mathbf{A}_{k}$ contain the differences in $\mathbf{A}_{m}$ from the tuned case, involving the mistuning patterns $\boldsymbol{\delta}_{j}$ and $\boldsymbol{\delta}_{k}$, respectively, and $\Delta \mathbf{q}_{j k}=\mathbf{q}_{j}-\mathbf{q}_{k}$. When the bottom partitions of Eq. (26) are combined with Eq. (20), the equivalent expression to Eq. (21) contains the unknown forcing, so that $\mathbf{q}$ cannot be determined directly. However, by subtracting two versions of the resulting equation to eliminate $\mathbf{F}^{\mathcal{S M A}}$, the differences in $\mathbf{q}$ can be determined based on differences in measurements $\overline{\mathbf{x}}_{\text {meas }}^{\mathbf{b}}$ :

$$
\begin{aligned}
& \left\{\begin{array}{c}
\Delta \tilde{\mathbf{q}}_{e_{j k}} \\
\boldsymbol{\Delta} \overline{\mathbf{q}}_{m_{j k}}
\end{array}\right\}=\left[\begin{array}{cc}
\mathbf{A}_{e} & \mathbf{A}_{e m}^{\mathbf{T}} \\
\mathbf{V}_{\text {meas }}
\end{array}\right]^{-1}\left\{\begin{array}{c}
0 \\
\overline{\mathbf{x}}_{\text {meas }_{j}}^{\mathbf{b}}-\overline{\mathbf{x}}_{\text {meas }_{k}}^{\mathbf{b}}
\end{array}\right\} \\
& (j, k)=(1,2),(2,3),(1,3)
\end{aligned}
$$

Here, $\boldsymbol{\Delta} \tilde{\mathbf{q}}_{e_{j k}}$ and $\boldsymbol{\Delta} \overline{\mathbf{q}}_{m_{j k}}$ are the partitions of $\boldsymbol{\Delta} \mathbf{q}_{j k}$, that is, $\Delta \tilde{\mathbf{q}}_{e_{j k}}=$ $\tilde{\mathbf{q}}_{e_{j}}-\tilde{\mathbf{q}}_{e_{k}}$ and $\boldsymbol{\Delta} \overline{\mathbf{q}}_{m_{j k}}=\overline{\mathbf{q}}_{m_{j}}-\overline{\mathbf{q}}_{m_{k}}$. Once $\Delta \mathbf{q}_{12}, \Delta \mathbf{q}_{23}$, and $\Delta \mathbf{q}_{13}$ are known, the bottom partitions of Eq. (28) can be used to solve for the unknown mistuning. In the absence of viscous damping,

$$
\Delta \mathbf{A}_{j}=(1+i G) \lambda_{b_{m}}\left[\operatorname{diag}_{n=1, \ldots, N} \delta_{j_{n}}\right]
$$

where $\delta_{j_{n}}$ is the $n$th element of mistuning pattern $\boldsymbol{\delta}_{j}$. When each $\boldsymbol{\Delta} \mathbf{A}_{i}$ is written in terms of $\boldsymbol{\delta}_{\mathbf{2}}$ and $\boldsymbol{\Delta} \boldsymbol{\delta}$ (using the relations $\boldsymbol{\delta}_{\mathbf{1}}=\boldsymbol{\delta}_{\mathbf{2}}-\boldsymbol{\Delta} \boldsymbol{\delta}$ and $\boldsymbol{\delta}_{\mathbf{3}}=\boldsymbol{\delta}_{\mathbf{2}}+\alpha \boldsymbol{\Delta} \boldsymbol{\delta}$ ), the bottom $N$ rows of Eq. (28) give

$$
\begin{aligned}
& {\left[\begin{array}{ll}
\mathbf{A}_{e m} & \mathbf{A}_{m}^{\text {tuned }}
\end{array}\right]\left\{\begin{array}{c}
\boldsymbol{\Delta} \tilde{\mathbf{q}}_{e_{12}} \\
\boldsymbol{\Delta} \overline{\mathbf{q}}_{m_{12}}
\end{array}\right\}+\left[\boldsymbol{\Delta} \mathbf{A}_{2}\right]\left\{\boldsymbol{\Delta} \overline{\mathbf{q}}_{m_{12}}\right\}} \\
& -\left[(1+i G) \lambda_{b_{m}}\right]\left[\operatorname{diag}_{n=1, \ldots, N} \quad \Delta \delta_{n}\right]\left\{\overline{\mathbf{q}}_{b_{1}}\right\}=\mathbf{0}
\end{aligned}
$$

and

$$
\begin{aligned}
& {\left[\begin{array}{ll}
\mathbf{A}_{e m} & \mathbf{A}_{m}^{\text {tuned }}
\end{array}\right]\left\{\begin{array}{c}
\boldsymbol{\Delta} \tilde{\mathbf{q}}_{e_{23}} \\
\boldsymbol{\Delta} \overline{\mathbf{q}}_{m_{23}}
\end{array}\right\}+\left[\boldsymbol{\Delta} \mathbf{A}_{2}\right]\left\{\boldsymbol{\Delta} \overline{\mathbf{q}}_{m_{23}}\right\}} \\
& -\left[\alpha(1+i G) \lambda_{b_{m}}\right]\left[\operatorname{diag}_{n=1, \ldots, N} \quad \Delta \delta_{n}\right]\left\{\overline{\mathbf{q}}_{m_{3}}\right\}=\mathbf{0}
\end{aligned}
$$

Equation (29) features terms containing $\boldsymbol{\Delta} \boldsymbol{\delta}$ multiplied by $\mathbf{q}_{1}$, whereas Eq. (30) has terms containing $\boldsymbol{\Delta} \boldsymbol{\delta}$ multiplied by $\mathbf{q}_{3}$. A linear combination of these two sets of equations creates equations in which $\Delta \delta$ terms are multiplied by $\Delta \mathbf{q}_{13}$ :

$$
\begin{aligned}
& {\left[\begin{array}{ll}
\mathbf{A}_{e m} & \mathbf{A}_{m}^{\text {tuned }}
\end{array}\right]\left\{\begin{array}{c}
\boldsymbol{\Delta} \tilde{\mathbf{q}}_{e_{12}}-\frac{1}{\alpha} \boldsymbol{\Delta} \tilde{\mathbf{q}}_{e_{23}} \\
\boldsymbol{\Delta} \overline{\mathbf{q}}_{m_{12}}-\frac{1}{\alpha} \boldsymbol{\Delta} \overline{\mathbf{q}}_{m_{23}}
\end{array}\right\}} \\
& +\left[(1+i G) \lambda_{b_{m}}\right]\left[\begin{array}{c}
\text { diag } \\
n=1, \ldots, N
\end{array} \quad \delta_{2_{n}}\right]\left\{\boldsymbol{\Delta} \overline{\mathbf{q}}_{m_{12}}-\frac{1}{\alpha} \boldsymbol{\Delta} \overline{\mathbf{q}}_{m_{23}}\right\} \\
& -\left[(1+i G) \lambda_{b_{m}}\right]\left[\begin{array}{c}
\text { diag } \\
n=1, \ldots, N
\end{array} \quad \Delta \delta_{n}\right]\left\{\boldsymbol{\Delta} \overline{\mathbf{q}}_{m_{13}}\right\}=\mathbf{0}
\end{aligned}
$$

Each row of this set of equations can then be solved for $\delta_{2_{n}}$, the $n$th element of the $\boldsymbol{\delta}_{2}$ pattern. The original, unknown mistuning is the pattern $\delta_{1}$, which can be recovered simply by subtracting the known change $\boldsymbol{\Delta} \delta$.

If viscous damping is not neglected, the matrix $\mathbf{A}$ includes the contributions of the viscous damping matrix $\mathbf{C}^{\mathcal{S M A}}$. The mistuned blade partition $\mathbf{A}_{m}$ thus includes the term $i \omega\left(2 \zeta \sqrt{\lambda_{b_{m}}}\right)$, where $\zeta$ is the damping ratio for the mistuned blade mode. Assuming that all $\delta$ are small (so that $\sqrt{1+\delta} \approx 1+\frac{1}{2} \delta$ ), the effect on Eq. (31) is merely to replace $\left[(1+i G) \lambda_{b_{m}}\right]$ with $\left[i \zeta \omega \sqrt{\lambda_{b_{m}}}+(1+i G) \lambda_{b_{m}}\right]$.

Sensitivity to small variations in measurement can be calculated with the same method as in the free response case, and a composite of several identifications can again ensure accurate mistuning identification for all blades. As before, measured frequencies near the blade-alone natural frequency produce results least sensitive to measurement variation. However, measurements are no longer limited to a finite number of natural frequencies; the forcing can be at any frequency desired, and so a greater number of sets of measurements is possible. The inputs to the forced response method are no longer the measurements themselves but the differences between sets of measurements, so that low values of $\beta$ are achieved not for blades with high vibration amplitude, but for blades with high differences between each of the three measured cases. Thus, the best additional patterns $[\boldsymbol{\Delta} \boldsymbol{\delta}$ and $(1+\alpha) \boldsymbol{\Delta} \boldsymbol{\delta}]$ are those that significantly alter the response at the measured frequencies. This can be achieved by focusing attention on frequency bands of high modal density, in which the additional mistuning pattern (which may be as simple as additional mistuning on a single blade) will affect several nearby modes. A measurement at a frequency at which multiple normal modes participate in the forced response will be altered most significantly when the participating mode shapes have changed and/ or the spacing between the natural frequencies has been increased or decreased.

The amplitude of the additional mistuning can be chosen by realizing that, to cause significant interaction between two neighboring modes, the shifts in frequency due to additional mistuning should be of the same order as the original spacing between modes. For systems that are very nearly tuned, normal modes occur in pairs of almost identical frequency, whereas systems with greater mistuning feature a wider spread in natural frequencies. Thus, the additional mistuning should be of at least the same degree as the original mistuning to cause a noticeable difference in system normal mode shapes (and thus in forced response behavior) between measurements.

\section{Experimental Validation}

To validate the mistuning identification techniques, an experiment was conducted using a blisk manufactured specifically for experimental studies of mistuning and precision machined to be as nearly tuned as possible. The techniques are applied both to the unmodified validation blisk, to confirm that any mistuning present is quite small, and to the blisk modified by a known mistuning pattern, to validate the techniques by successful identification of that pattern.

\section{A. Experimental Setup}

Figure 1 shows a photograph of the experimental setup, which includes an integrally bladed disk (the validation blisk, top right), two systems for measuring the response (top left and bottom), and an excitation system consisting of small speakers, one positioned behind each blade of the blisk. The excitation and measurement systems are all noncontacting to avoid unintended sources of mistuning.

The speakers are driven by 24 independent signals, so that single blade excitation is possible by driving one speaker, whereas more complex patterns of excitation are possible by driving all speakers at the same frequency but at different amplitudes or phases. By driving each speaker at the same amplitude but at a precise phase lag from the adjacent speaker, a rotating, engine-order-type excitation can be created that mimics the effect of rotating the blisk past an arbitrary number of stationary excitation sources. An electronic specklepattern interferometry system is used to observe the response of the entire blisk at once, allowing rapid characterization of the response patterns of the blisk. Quantitative measurements, those used as input to the mistuning identification techniques, are gathered using a single-point laser vibrometer, which is mounted on a two-stage computer-controlled linear traverse. A detailed description of the experimental setup, including excitation and measurement systems, is given in [35].

The validation blisk is a 24 bladed disk manufactured from a single piece of 1117 steel. Each blade is 2.000-in. $(50.80-\mathrm{mm})$ long, and the disk has an outer radius of $5.900 \mathrm{in}$. (149.86 mm), for a total blisk diameter of $15.80 \mathrm{in} .(401.3 \mathrm{~mm})$. The blades are of rectangular cross section, 0.2500-in. (6.35-mm) thick and 1.6480-in. (41.859-mm) wide, and are set at a $30 \mathrm{deg}$ angle out of the plane of the disk. The blades are somewhat thick compared to the blades of industrial rotors; the thickness was chosen to minimize the impact of small 


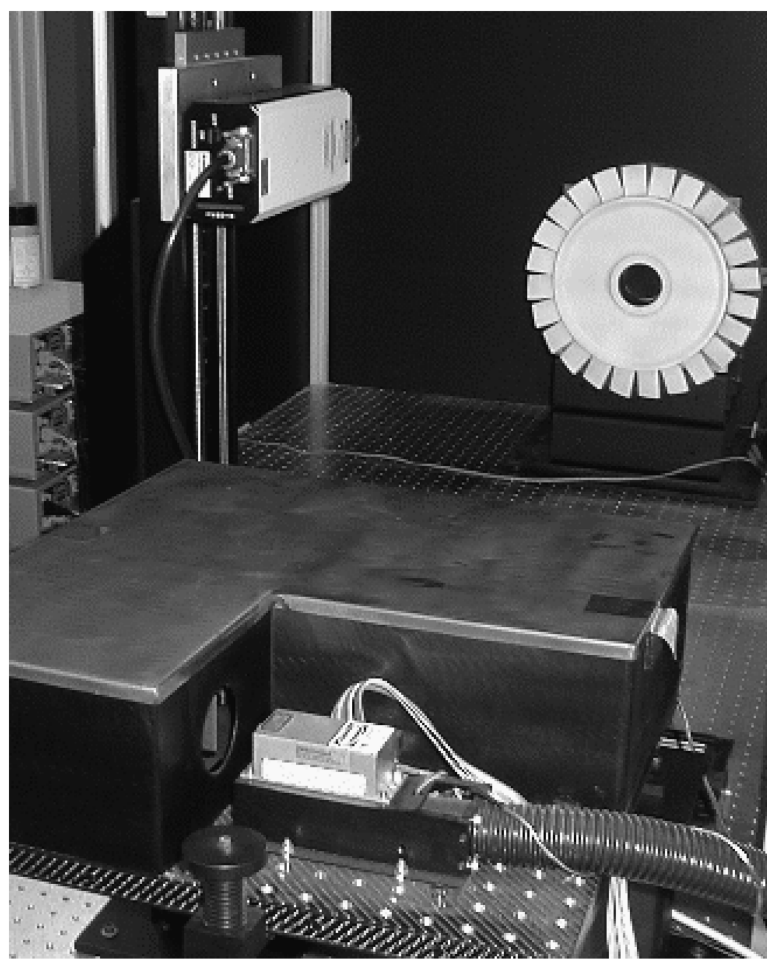

Fig. 1 Photograph of experimental setup.

variations in blade thickness on the natural frequencies of the blades. The tolerance on this critical dimension is less than 0.0005 in. $(0.013 \mathrm{~mm})$, so that the blade thickness varies by less than $0.2 \%$, resulting in a blisk that is very nearly tuned. Tolerances on other dimensions are better than 0.005 in. $(0.127 \mathrm{~mm})$.

There is a 0.1875 -in. $(4.76-\mathrm{mm})$ fillet at the blade root, where the blades protrude from the disk's outer drum. The outer drum is $1.600-$ in. (40.64-mm) thick in the axial direction, and 0.250-in. (6.35-mm) wide in the radial direction; the disk then tapers inward at a $45 \mathrm{deg}$ angle until it reaches its nominal thickness of $0.750 \mathrm{in.}(19.05 \mathrm{~mm})$. The center of the disk is a hole, 1.500 in. $(38.10 \mathrm{~mm})$ in radius, surrounded by a 0.500 -in. (12.70-mm) wide inner drum. On one side of the disk, the drum extends into a mounting flange containing a number of threaded holes to allow the blisk to be bolted to its support.

The finite element model (FEM) of the validation blisk is shown in Fig. 2. Each blade has 1254 DOF, while the disk has 4518 DOF per sector, and each disk-blade interface has $108 \mathrm{DOF}$, for a total of $141,120 \mathrm{DOF}$ in the entire blisk. All 108 interface DOF are retained in the CMS model, in addition, the first 15 blade normal modes and the first 15 disk modes are retained, for a total of 138 modes for each of the 24 harmonics, resulting in 3312 DOF in the intermediate

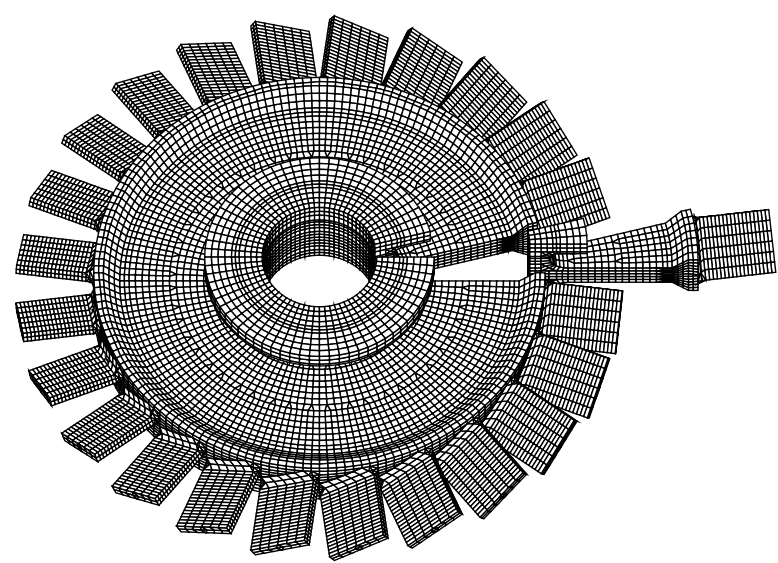

Fig. 2 Finite element mesh of the validation blisk with one sector cut out to reveal cross section. model. This results in frequency error relative to the original FEM model on the order $10^{-6}$ or better for all modes in the mode family studied in this experiment, and $10^{-3}$ or better for all of the first 95 modes of the system. (Retaining only 10 disk modes resulted in an order of magnitude increase in error for several modes, whereas retaining 20 disk or blade modes did not result in significant improvement.) The SMA model maintains much closer fidelity to the CMS model than the CMS does to the original FEM; retaining 49 secondary modes per harmonic results in frequencies that differ from the CMS model frequencies only because of numerical roundoff error. To minimize the final model size, 29 secondary modes were retained per harmonic for this study, resulting in frequency error on the order $10^{-6}$ relative to the intermediate model for the system modes of interest. These modes, plus the single mistuned blade mode (in this case, the first blade flexural mode), result in a total of 720 DOF in the final model.

Figure 3 shows the free vibration natural frequencies of the blisk versus the number of nodal diameters in the corresponding mode shape, as predicted by the full finite element model. The mode family of interest for this validation is the first blade flexural mode family (dominated by out-of-plane bending of the blades) near $2100 \mathrm{~Hz}$. For these modes, the differences between natural frequencies predicted by the finite element model and the reduced-order model are on the order of $0.001 \mathrm{~Hz}$. Experimental measurements indicate the actual natural frequencies of the validation blisk differ from those predicted by less than $0.3 \%$ (about $6 \mathrm{~Hz}$ ) for the modes of interest.

\section{B. Free Response Method Validation \\ 1. "Tuned" Case}

By driving only one speaker at a time behind a blade chosen to produce strong response in one mode but little or no response in modes nearby in frequency, single modes of the blisk were excited in isolation. This is possible due to the extremely low damping present, resulting in very narrow resonant peaks (the structural damping coefficient $G$ is approximately 0.00015 , based on a log-decrement

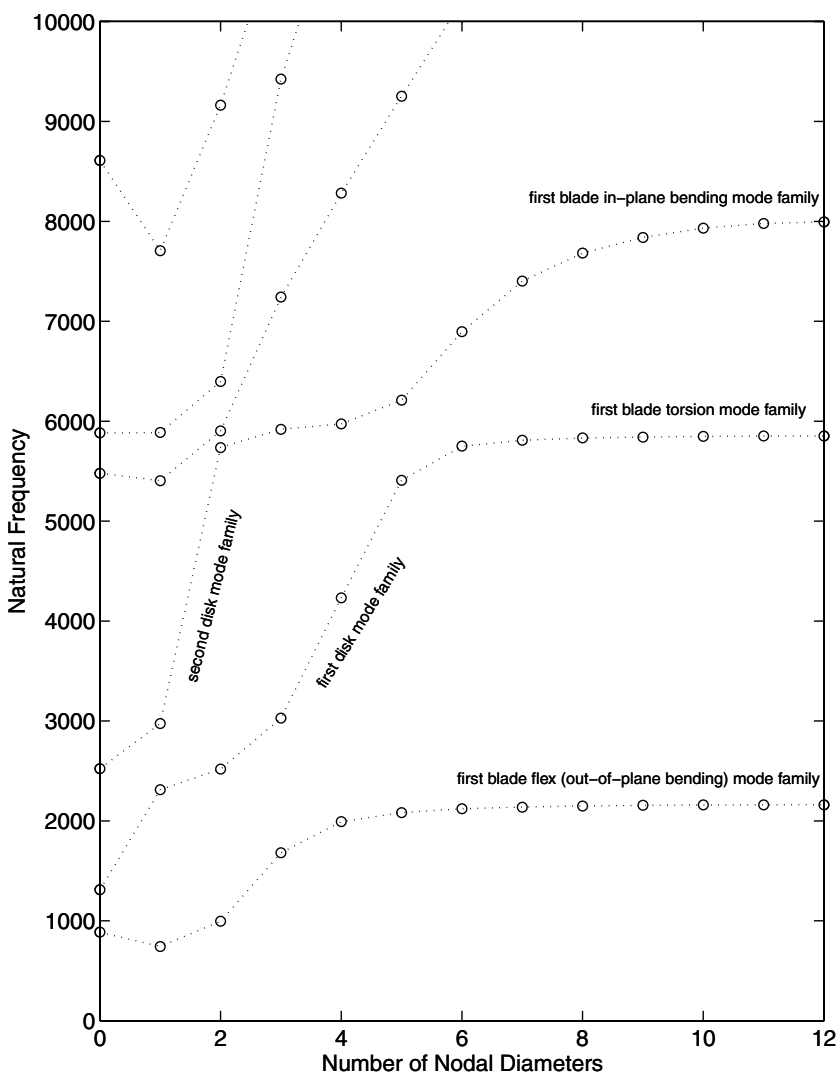

Fig. 3 Validation blisk natural frequencies vs number of nodal diameters in the corresponding mode shapes. 
measurement of the validation blisk). A number of mode shapes in the first blade flexural family of the nonmistuned blisk were measured and input to the free response mistuning identification algorithm. Figure 4 shows the identified blade mistuning parameters $\delta_{n}$ (for $\left.n=1, \ldots, \overline{2} 4\right)$, which represent the deviation of each blade's modal stiffness from the stiffness of the first flexural mode of the nominal, tuned blade. The figure shows all raw $\delta_{n}$ results with corresponding error magnification factor $\beta_{n}$ less than 1.25 , a cutoff value selected to be as low as possible while being high enough that at least one $\delta_{n}$ was retained for each blade. Figure 4 also shows the average value for each blade, taken to be the composite identified blade mistuning. The average mistuning across all 24 blades is just less than $0.5 \%$, indicating that the nominal blade frequency in the reduced-order model is quite close to the actual mean blade frequency. The standard deviation is $0.33 \%$, and so the blisk is quite nearly tuned. Because the blade natural frequency is the square root of the blade eigenvalue, the deviation in frequency is approximately $0.17 \%$.

Confidence that the mistuning pattern has been correctly identified can be gained by calculating the theoretical mode shapes of a blisk mistuned with the identified pattern. Figure 5 shows the measured amplitude at the tip of each blade for several modes and compares these shapes with the theoretically predicted mode shapes. These

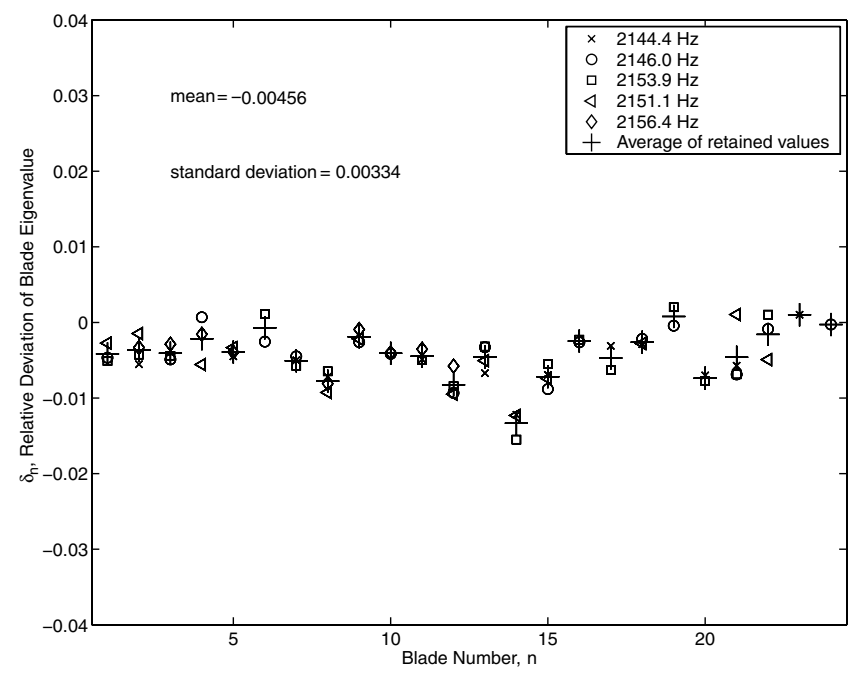

Fig. 4 Mistuning identification of tuned validation blisk using free response measurements.
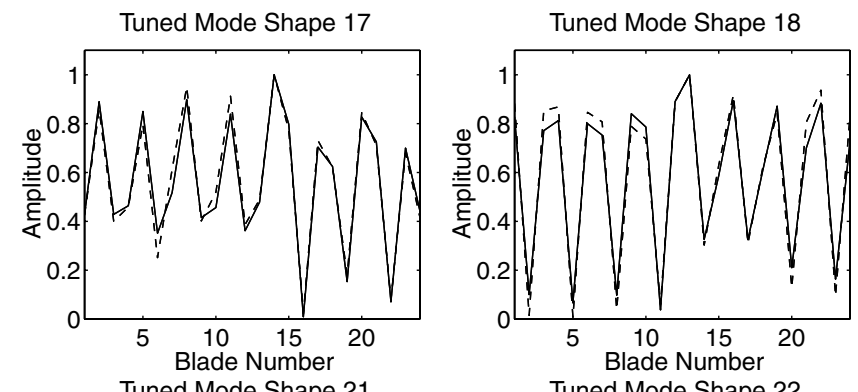

Tuned Mode Shape 21
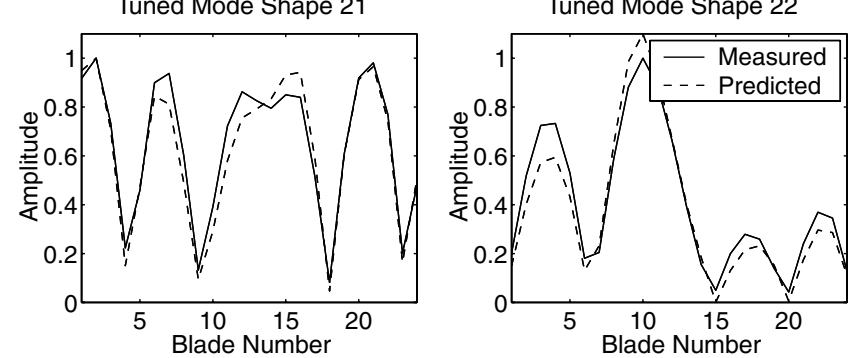

Fig. 5 Comparison of measured and predicted free vibration mode shapes for tuned validation blisk. modes are typical; there is, in general, excellent agreement between measurements and predictions.

\section{Mistuned Case}

To validate the mistuning identification method, a known mistuning pattern was added to the blisk. Small pieces of lead were glued to the tips of six of the blades to decrease the blade eigenvalue corresponding to the first flexural mode of the blades. A $1.0 \%$ decrease was implemented on blade 10 by adding a $0.23 \mathrm{~g}$ lead piece, $2.0 \%$ decreases were given to blades 3,8 , and 18 by adding $0.47 \mathrm{~g}$, and the addition of $0.71 \mathrm{~g}$ decreased the eigenvalues of blades 4 and 22 by $3.0 \%$.

Measurements were again made of the blisk modes in the first blade flex family. The results of the mistuning identification algorithm are shown in Fig. 6: in this case, $\delta$ s with corresponding $\beta$ less than 0.8 were retained. The average prediction for each blade is compared with the expected value based on the average tuned case identification plus the effect of the additional lead weights.

The match between tuned and mistuned cases for the unmodified blades is quite good. The identification technique also correctly predicts which blades have been altered, but overpredicts the amount of mistuning by $10-30 \%$. This is due to the assumption in the reduced-order model that mistuning occurs only as a change in blade modal stiffness (eigenvalue). This is often an acceptable assumption in cases in which mistuning is due to minor variations in blade geometry or material properties. However, the case of additional mass at the blade tip represents an extreme case of mistuning in which the blade mode shape (eigenvector) is also significantly affected. The effect of the changes in blade mode shape can be accounted for by using this mistuning identification as a first iteration. New blade component modes are generated in the CMS model for the blades with significant mistuning, and the algorithm is run again to generate a new, more accurate mistuning pattern. Figure 7 shows the results of this adjustment. Note the significantly improved identification of the mistuning levels on the mistuned blades: $3,4,8,10,18$, and 22 .

Figure 8 shows a comparison of the theoretical mode shapes predicted $\bar{b}$ ased on the identified mistuning pattern and the actual, measured mode shapes. Again, the excellent agreement, observed for both extended and localized modes, gives a measure of added confidence in the success of the mistuning identification technique.

\section{Forced Response Method Validation}

The free response mistuning identification method works quite well, but requires that the modal density and damping are such that individual mode shapes can be excited in isolation. Mistuning

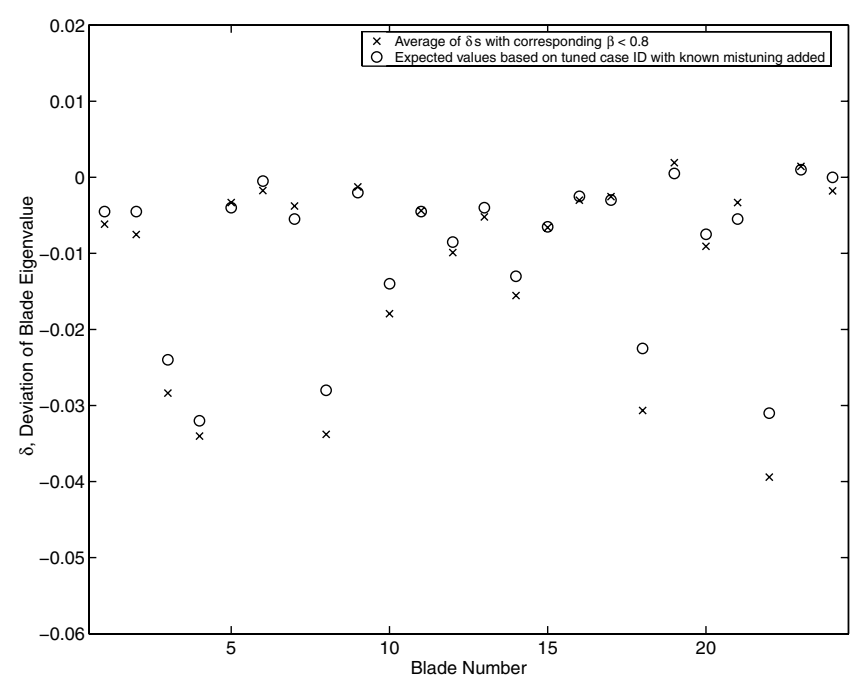

Fig. 6 Average mistuning identification of mistuned validation blisk using free response measurements compared with expected value based on tuned case identification with known added mistuning. 


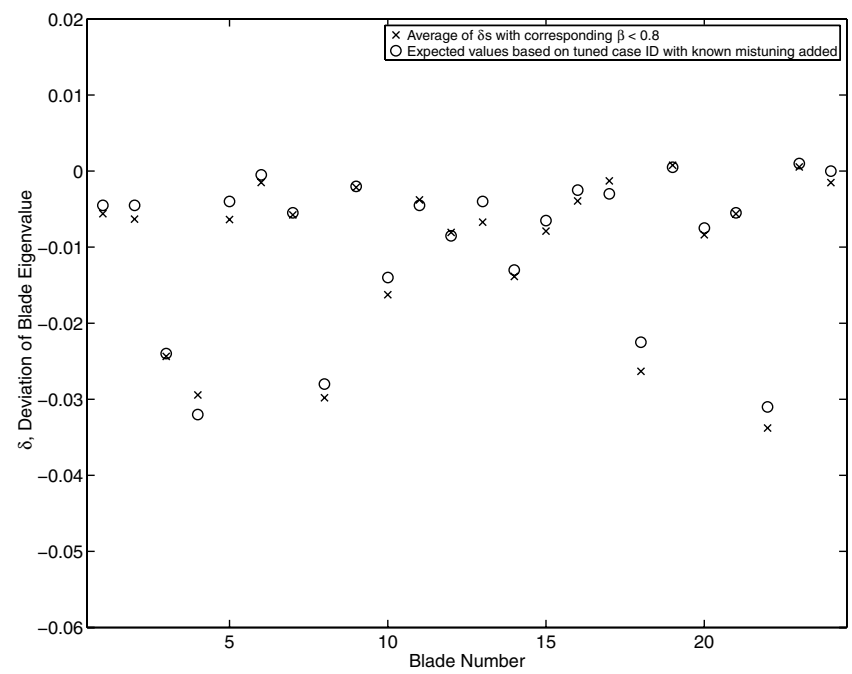

Fig. 7 Average free response mistuned case identification using adjusted mode shapes for blades $3,4,8,10,18$, and 22 .
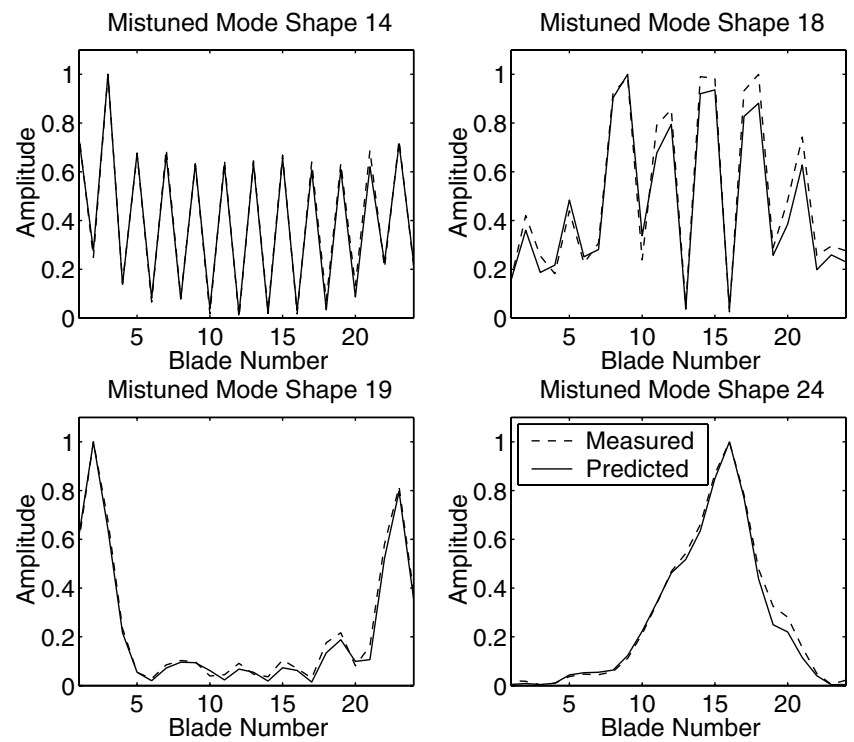

Fig. 8 Comparison of measured and predicted mode shapes for mistuned validation blisk.

identification based on forced response measurements does not have this requirement, as it does not matter if the response at a particular frequency consists of contributions from several different modes. However, the forced response method does entail a greater number of experimental measurements and requires careful selection of the additional mistuning patterns $\boldsymbol{\Delta} \boldsymbol{\delta}$ and $(1+\alpha) \boldsymbol{\Delta} \boldsymbol{\delta}$ (see Sec. IV).

\section{Tuned Case}

For the nonmistuned blisk, blade 23 was chosen to receive additional mistuning due to the fact that it has a strong response in several of the modes in the frequency range of interest. Forced response amplitude and phase measurements were taken of the unmodified blisk at a large number of excitation frequencies in the area of high modal density between 2100 and $2160 \mathrm{~Hz}$. The measurements were then repeated with $0.23 \mathrm{~g}$ and then $0.47 \mathrm{~g}$ lead weights affixed to tip of blade 23 , corresponding to a $1.0 \%$ and $2.0 \%$ decrease in that blade's eigenvalue for the $\boldsymbol{\Delta} \boldsymbol{\delta}$ and $(1+\alpha) \boldsymbol{\Delta} \boldsymbol{\delta}$ patterns, respectively (in this case, $\alpha=1$ and $\Delta \delta_{n}=-0.01$ for $n=23$ and zero for all other $n$ ). Figure 9 shows the results of the mistuning identification using the forced response method: $\delta \mathrm{s}$ are retained that have corresponding error magnification $\beta$ less than 1.35 , and the average value for each blade is compared with the average value from the free response identification. In general, there

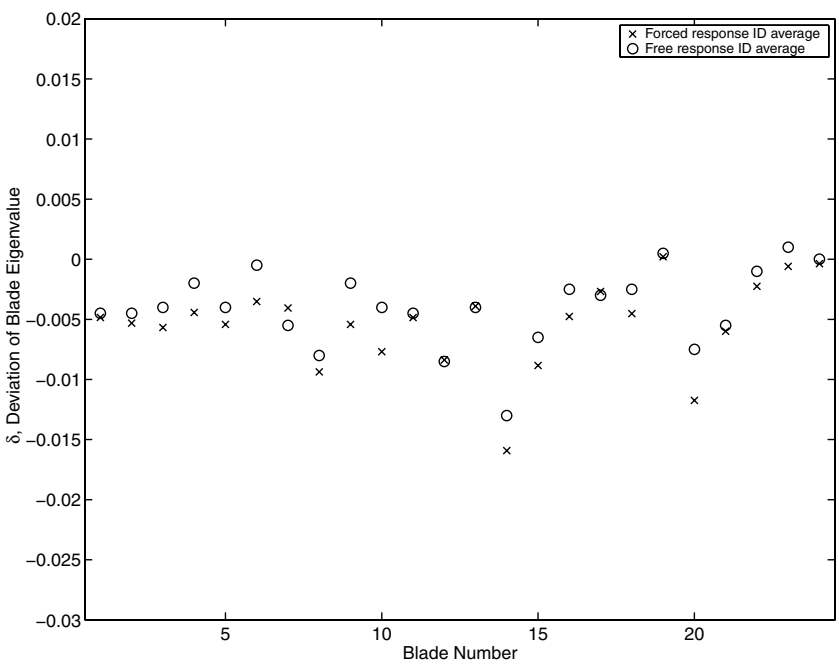

Fig. 9 Comparison of average mistuning identification of tuned validation blisk using free and forced response measurements.

is good agreement between the two methods; the largest discrepancy is less than 0.005 .

\section{Mistuned Case}

After mistuning the blisk with the pattern described in Sec. V.B.2, blade 12 was chosen to receive the additional mistuning, due to its strong response at several resonant peaks, and the additional mistuning was increased to 2.0 and $4.0 \%$ due to the larger amount of mistuning to be identified (thus, $\Delta \delta_{n}=-0.02$ for $n=12$ and zero for all other $n$, and $\alpha$ is again one). Figure 10 shows the results of the identification, retaining all $\delta$ s with corresponding $\beta$ less than 2.5 . The average values for each blade are compared with the expected value based on the tuned case plus the known added mistuning (in this case, the forced response tuned case is used). Like the free response method, the forced response method predicts values for the unmodified blades that are consistent with the tuned case identifications, but overpredicts somewhat the amount of mistuning on the modified blades. Once again, this is due to the fact that adding mass to the blade tips is an extreme form of mistuning that has a nonnegligible effect on the blade mode shapes, and the identification is improved by including the modified mode shapes in the CMS model.

Comparison of Figs. 6 and 10 shows that, for the validation blisk, both free and forced response methods recover the added known

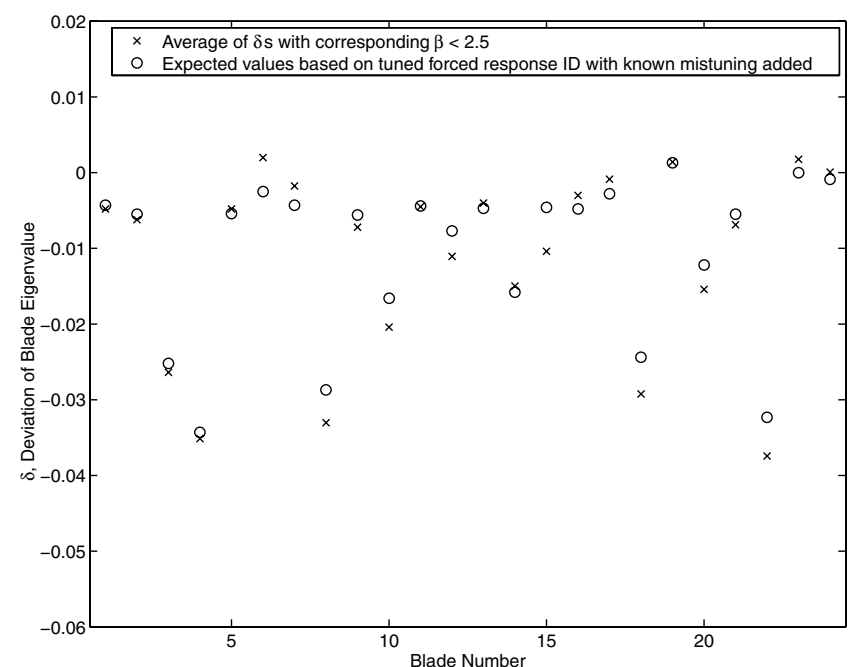

Fig. 10 Average mistuning identification of mistuned validation blisk using forced response measurements compared with expected value based on tuned forced response identification with known added mistuning. 
mistuning with a similar degree of accuracy. For a blisk with higher damping or higher modal density, such that free vibration mode shapes were more difficult to isolate experimentally, one would expect the accuracy of the free response method to be compromised, while the forced response method would be unaffected.

\section{Conclusions}

The two methods presented here for identifying individual blade mistuning using measurements of the response of the entire structure are especially suitable for blisks, in which individual blades cannot be tested in isolation. The first method, which makes use of measurements of one or more free vibration mode shapes, is straightforward in application, and is suitable for specimens with very low damping or relatively low modal density. The second method, which makes use of measurements of the steady-state forced response of the bladed disk at one or more excitation frequencies, requires the user to carefully select excitation frequencies and to add known mistuning to the specimen when performing certain measurements, but is extremely powerful because any harmonic forcing of the specimen can be used, even if the pattern and strength of the forcing are unknown. It is suitable for specimens in which high modal density or relatively high damping causes resonant peaks to overlap and merge, making isolation of normal modes more difficult.

Experimental validation of both methods was presented using results obtained from measurements of a specially designed blisk. The free and forced response methods produced consistent results when identifying the small mistuning present in the unmodified blisk. This mistuning pattern was validated by numerically predicting the mode shapes due to the identified pattern and comparing them to the measured shapes. A known pattern of mistuning was then added to the specimen, and both identification methods successfully identified the blades that were altered and the degree to which the modal stiffnesses were changed.

\section{Acknowledgments}

This work was supported by grants from the U.S. Air Force Office of Scientific Research and NASA John H. Glenn Research Center.

\section{References}

[1] Castanier, M. P., and Pierre, C., "Modeling and Analysis of Mistuned Bladed Disk Vibration: Status and Emerging Directions," Journal of Propulsion and Power, Vol. 22, No. 2, 2006, pp. 384-396. doi:10.2514/1.16345

[2] Irretier, H., "Spectral Analysis of Mistuned Bladed Disk Assemblies by Component Mode Synthesis," Vibrations of Bladed Disk Assemblies, Proceedings of the ASME 9th Biennial Conference on Mechanical Vibration and Noise, American Society of Mechanical Engineers, Fairfield, NJ, 1983, pp. 115-125.

[3] Zheng, Z.-C., and Wang, F.-R., "Dynamic Analysis of Blade Groups Using Component Mode Synthesis," Vibrations of Blades and Bladed Disk Assemblies, Proceedings of the ASME 10th Biennial Conference on Mechanical Vibration and Noise, American Society of Mechanical Engineers, Fairfield, NJ, 1985, pp. 97-103.

[4] Kruse, M. J., and Pierre, C., "Forced Response of Mistuned Bladed Disks Using Reduced-Order Modeling," Proceedings of the 37th AIAA/ ASME Structures, Structural Dynamics, and Materials Conference, AIAA, Reston, VA, 1996, pp. 1938-1950.

[5] Castanier, M. P., Óttarsson, G., and Pierre, C., "A Reduced-Order Modeling Technique for Mistuned Bladed Disks," Journal of Vibration and Acoustics, Vol. 119, No. 3, 1997, pp. 439-447. doi: $10.1115 / 1.2889743$

[6] Yang, M.-T., and Griffin, J. H., "A Reduced Order Approach for the Vibration of Mistuned Bladed Disk Assemblies," Journal of Engineering for Gas Turbines and Power, Vol. 119, No. 1, 1997, pp. $161-167$. doi:10.1115/1.2815542

[7] Yang, M.-T., and Griffin, J. H., "A Reduced Order Model of Mistuning Using a Subset of Nominal System Modes," Journal of Engineering for Gas Turbines and Power, Vol. 123, No. 4, 2001, pp. 893-900. doi:10.1115/1.1385197

[8] Bladh, R., Castanier, M. P., and Pierre, C., "Reduced Order Modeling and Efficient Forced Response Statistics Prediction for Mistuned
Bladed Disks," Proceedings of The 3rd National Turbine Engine High Cycle Fatigue Conference, Universal Technology Corp., Dayton, $\mathrm{OH}$, 1998.

[9] Bladh, R., Castanier, M. P., and Pierre, C., "Reduced Order Modeling and Vibration Analysis of Mistuned Bladed Disk Assemblies with Shrouds," Journal of Engineering for Gas Turbines and Power, Vol. 121, No. 3, 1999, pp. 515-522. doi:10.1115/1.2818503

[10] Bladh, R., Castanier, M. P., and Pierre, C., "Component-Mode-Based Reduced Order Modeling Techniques for Mistuned Bladed Disks, Part I: Theoretical Models," Journal of Engineering for Gas Turbines and Power, Vol. 123, No. 1, 2001, pp. 89-99. doi: $10.1115 / 1.1338947$

[11] Bladh, R., Castanier, M. P., and Pierre, C., "Component-Mode-Based Reduced Order Modeling Techniques for Mistuned Bladed Disks, Part II: Application," Journal of Engineering for Gas Turbines and Power, Vol. 123, No. 1, 2001, pp. 100-108. doi: $10.1115 / 1.1338948$

[12] Feiner, D. M., and Griffin, J. H., "A Fundamental Model of Mistuning for a Single Family of Modes," Journal of Turbomachinery, Vol. 124, No. 4, 2002, pp. 597-605. doi:10.1115/1.1508384

[13] Lim, S., Bladh, R., Castanier, M. P., and Pierre, C., "A Compact, Generalized Component Mode Mistuning Representation for Modeling Bladed Disk Vibration," Collection of Technical Papers: AIAA/ASME/ ASCE/AHS/ASC Structures, Structural Dynamics and Materials Conference, Vol. 2, AIAA, Reston, VA, 2003, pp. 1359-1380.

[14] Lim, S., Bladh, R., Castanier, M. P., and Pierre, C., "Compact, Generalized Component Mode Mistuning Representation for Modeling Bladed Disk Vibration," AIAA Journal, Vol. 45, No. 9, 2007, pp. 22852298.

doi:10.2514/1.13172

[15] Mignolet, M. P., and Rivas-Guerra, A., "Identification of Mistuning Characteristics of Bladed Disks from Free Response Data, Part I," Journal of Engineering for Gas Turbines and Power, Vol. 123, No. 2, 2001, pp. 395-403. doi: $10.1115 / 1.1338949$

[16] Mignolet, M. P., and Rivas-Guerra, A., "Identification of Mistuning Characteristics of Bladed Disks from Free Response Data, Part II," Journal of Engineering for Gas Turbines and Power, Vol. 123, No. 2, 2001, pp. 404-411.

doi:10.1115/1.1338950

[17] Judge, J. A., Pierre, C., and Ceccio, S. L., "Experimental Identification of Mistuning in Bladed Disks," Proceedings of the 6th National Turbine Engine High Cycle Fatigue Conference, Universal Technology Corp., Dayton, OH, 2002.

[18] Judge, J., Pierre, C., and Ceccio, S. L., "Experimental Validation of Mistuning Identification Techniques and Vibration Predictions in Bladed Disks," Proceedings of the 2001 CEAS/AAIA/AIAE International Forum on Aeroelasticity and Structural Dynamics, Vol. 2, AlfaSur, Madrid, 2001, pp. 89-98, ISBN 84-931375-6-1.

[19] Judge, J. A., Pierre, C., and Ceccio, S. L., "Mistuning Identification in Bladed Disks," Proceedings of the International Conference on Structural Dynamics Modelling, 2002.

[20] Mignolet, M. P., and Lin, C.-C., "Identification of Structural Parameters in Mistuned Bladed Disks," Journal of Vibration and Acoustics, Vol. 119, No. 3, 1997, pp. 428-438. doi: $10.1115 / 1.2889742$

[21] Pichot, F., Thouverez, F., Jezequel, L., and Seinturier, E., "Mistuning Parameters Identification of a Bladed Disk," Key Engineering Materials: Damage Assessment of Structures; DAMAS 2001, Vols. 204-205, TransTech Publ., Switzerland, 2001, pp. 123-132.

[22] Feiner, D. M., and Griffin, J. H., "A Completely Experimental Method of Mistuning Identification in Integrally Bladed Rotors," Proceedings of the 8th National Turbine Engine High Cycle Fatigue Conference, Universal Technology Corp., Dayton, OH, 2003, pp. 1.1-1.13.

[23] Kim, N. E., and Griffin, J. H., "System Identification in Higher Modal Density Regions of Bladed Disks," Proceedings of the 8th National Turbine Engine High Cycle Fatigue Conference, Universal Technology Corp., Dayton, OH, 2003, pp. 1.68-1.82.

[24] Lim, S.-H., Pierre, C., and Castanier, M. P., "System Identification in Higher Modal Density Regions of Bladed Disks," Proceedings of the 9th National Turbine Engine High Cycle Fatigue Conference, Universal Technology Corp., Dayton, OH, 2004.

[25] Feiner, D., and Griffin, J., "Mistuning Identification of Bladed Disks Using a Fundamental Mistuning Model, Part I: Theory," Journal of Turbomachinery, Vol. 126, No. 1, 2004, pp. 150-158. doi:10.1115/1.1643913

[26] Feiner, D., and Griffin, J., "Mistuning Identification of Bladed Disks 
Using a Fundamental Mistuning Model, Part II: Application,” Journal of Turbomachinery, Vol. 126, No. 1, 2004, pp. 159-165. doi:10.1115/1.1643914

[27] Li, J., Pierre, C., and Ceccio, S. L., "Validation of a New Technique for Mistuning Identification and Model Updating Based on Experimental Results for an Advanced Bladed Disk Prototype," Evaluation, Control and Prevention of High Cycle Fatigue in Gas Turbine Engines for Land, Sea and Air Vehicles, NATO Research and Technology Organization RTO-MP-AVT-121, 2005, pp. 36-1-36-16.

[28] Pichot, F., Laxalde, D., Sinou, J. J., Thouverez, F., and Lombard, J. P., "Mistuning Identification for Industrial Blisks Based on the Best Achievable Eigenvector," Computers \& Structures, Vol. 84, Nos. 2930, 2006, pp. 2033-2049.

doi:10.1016/j.compstruc.2006.08.022

[29] Laxalde, D., Thouverez, F., Sinou, J.-J., Lombard, J.-P., and Baumhauer, S., "Mistuning Identification and Model Updating of an Industrial Blisk," International Journal of Rotating Machinery, Vol. 2007, No. 1, 2007, pp. 17289. doi: $10.1155 / 2007 / 17289$

[30] Song, S. H., Castanier, M. P., and Pierre, C., "System Identification of Multistage Turbine Engine Rotors," Proceedings of the ASME Turbo Expo, Vol. 5, American Society of Mechanical Engineers, Fairfield, NJ, 2007, pp. 569-582.
[31] Madden, A. C., Castanier, M. P., and Epureanu, B. I., "Reduced-Order Model Construction Procedure for Robust Mistuning Identification of Blisks," Proceedings of the 49th AIAA/ASME/ASCE/AHS/ASC Structures, Structural Dynamics, and Materials Conference, AIAA, Reston, VA, 2008.

[32] Craig, R. R., and Bampton, M. C. C., "Coupling of Substructures for Dynamics Analyses," AIAA Journal, Vol. 6, No. 7, 1968, pp. 13131319. doi: $10.2514 / 3.4741$

[33] Tan, Y.-C., Castanier, M. P., and Pierre, C., "Modal Approximations of Power Flow Between Coupled Component Structures," Proceedings of the Sixth International Congress on Sound and Vibration, Univ. of Denmark, Lyngby, Denmark, 1999.

[34] Davis, P. J., Circulant Matrices, Wiley-Interscience, New York, 1979.

[35] Judge, J. A., Pierre, C., and Ceccio, S. L., "Traveling-Wave Excitation and Optical Measurement Techniques for Non-Contacting Investigation of Bladed Disk Dynamics," Shock and Vibration Digest, Vol. 35, No. 3, 2003, pp. 183-190. doi: $10.1177 / 0583102403035003002$

R. Kapania Associate Editor 